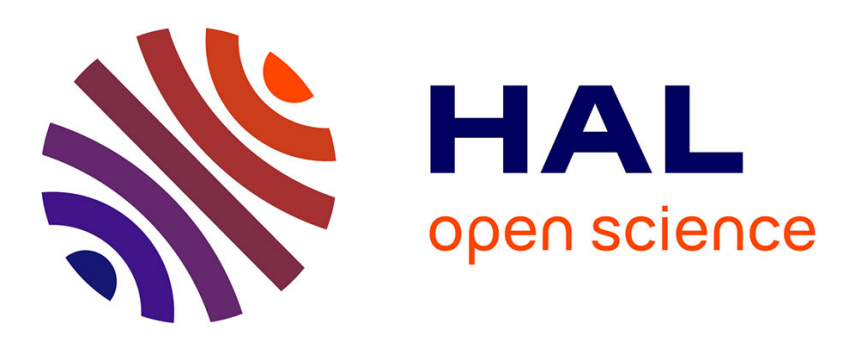

\title{
Plasma-Induced Polymerizations: A New Synthetic Entry in Liquid Crystal Elastomer Actuators
}

\author{
Bin Ni, Mengxue X Zhang, Cédric M Guyon, Patrick Keller, Michael H
} Tatoulian, Min-Hui Li

\section{- To cite this version:}

Bin Ni, Mengxue X Zhang, Cédric M Guyon, Patrick Keller, Michael H Tatoulian, et al.. PlasmaInduced Polymerizations: A New Synthetic Entry in Liquid Crystal Elastomer Actuators. Macromolecular Rapid Communications, 2020, 41 (19), pp.2000385. 10.1002/marc.202000385 . hal03051790

\section{HAL Id: hal-03051790 \\ https://hal.science/hal-03051790}

Submitted on 22 Dec 2020

HAL is a multi-disciplinary open access archive for the deposit and dissemination of scientific research documents, whether they are published or not. The documents may come from teaching and research institutions in France or abroad, or from public or private research centers.
L'archive ouverte pluridisciplinaire HAL, est destinée au dépôt et à la diffusion de documents scientifiques de niveau recherche, publiés ou non, émanant des établissements d'enseignement et de recherche français ou étrangers, des laboratoires publics ou privés. 


\section{WILEY-VCH}

Plasma-induced polymerizations: a new synthetic entry in liquid crystal elastomer actuators

Bin Ni, ${ }^{a}$ Mengxue Zhang, ${ }^{a}$ Cedric Guyon, ${ }^{a}$ Patrick Keller, ${ }^{* b}$ Michael Tatoulian, ${ }^{*}, a$ and Min-Hui Li*a

B. Ni, Dr. M. X. Zhang, Dr. C. Guyon, Prof. M. Tatoulian, Dr. M.H. Li,

Chimie ParisTech, PSL University Paris, CNRS, Institut de Recherche de Chimie Paris, UMR8247, 11 rue Pierre et Marie Curie, 75005 Paris, France.

E-mail: michael.tatoulian@chimieparistech.psl.eu,min-hui.li@chimieparistech.psl.eu

Dr. P. Keller

Institut Curie, Laboratoire Physico-Chimie Curie, UMR168, 11 rue Pierre et Marie Curie, 75005 Paris, France

E-mail: patrick.keller@curie.fr

Keywords: Liquid crystal elastomer, Plasma, Magnetic field, Thermomechanical, Soft robots.

The research on soft actuators including liquid crystal elastomers (LCEs) becomes more and more appealing at a time when the expansion of artificial systems is blooming. Among the various LCEs actuators, the bending deformation is often in the origin of many actuation modes. Here a new strategy with plasma technology was developed to prepare single-layer main-chain LCEs with thermally actuated bending and contraction deformations. Two distinct reactions, plasma polymerization and plasma-induced photopolymerization, were used to polymerize in one step the nematic monomer mixture aligned by magnetic field. The plasma polymerization formed crosslinked but disoriented structures at the surface of the LCE film, while the plasmainduced photopolymerization produced aligned LCE structure in the bulk. The actuation behaviors (bending and/or contraction) of LCE films could be adjusted by plasma power, reaction time, and sample thickness. Soft robots like crawling walker and flower mimic were built by LCE films with bending actuation. 


\section{WILEY-VCH}

\section{Introduction}

At a time when the expansion of artificial systems through our society is rapidly blooming, the research on soft actuators becomes more and more appealing and necessary. ${ }^{[1,2]}$ Liquid crystal elastomers (LCEs), as a type of stimuli-responsive materials, ${ }^{[3-7]}$ exhibit outstanding reversible shape-memory properties when exposed to external stimulations. ${ }^{[8-13]}$ Since P. G. De Gennes ${ }^{[14]}$ predicted the possibility of making LCE actuators in 1975, the continuous research and developments on LCEs attracted considerable attention and found potential applications as artificial muscles, ${ }^{[15-17]}$ smart sensors, ${ }^{[18-20]}$ motors $^{[21]}$ and actuators. ${ }^{[22-24]}$ Finkelmann ${ }^{[25]}$ pioneered the two-step mechanical stretch and hydrosilylation method to obtain aligned LCEs with reversible contraction and extension deformation. On the other hand, radiation-induced polymerizations such as photopolymerization ${ }^{[26,27]}$ are the most popular methods. Being combined with different alignment procedures like magnetic alignment, ${ }^{[8,28]}$ surface patterning technology, ${ }^{[29,}{ }^{30]}$ microfluidic alignment ${ }^{[12]}$ and microchannel alignment, ${ }^{[31,}{ }^{32]}$ photopolymerization has contributed widely to the development of the LCEs, especially those with sophisticated three-dimensional (3D) shape deformations. More recently, another radiation-induced polymerization, electron-beam polymerization ${ }^{[33,34]}$ has also been used to prepare LCEs.

Among the various LCEs actuators, the bending deformation is often in the origin of many actuation modes. A common strategy to achieve bending deformation used bilayer or multilayer heterogeneous materials, ${ }^{[9,33,35,36]}$ similar to the bimorph concept. However, this strategy might suffer from delamination or interfacial sliding during repeating actuations. Thus, it would be desirable to have access to the bending deformation with homogeneous single-layer materials. For this purpose, spatially modulated mesogens' orientations, such as twist, ${ }^{[37]}$ splay, ${ }^{[38]}$ and azimuthal or radial alignments, ${ }^{[29]}$ were designed in single-layer using surface anchoring technologies for alignment. These single-layer LCE actuators were finally obtained by photopolymerization, which exhibited bending or others sophisticated deformations. However, 


\section{WILEY-VCH}

surface anchoring technologies have limitation in sample thickness, and the reported LCE films have thickness $<200 \mu \mathrm{m}$. On the other hand, sequential or gradient crosslinking, ${ }^{[22], ~}{ }^{[39,40]} 3 \mathrm{D}$ printing, ${ }^{[41]}$ and photolithography ${ }^{[42,43]}$ were also employed to make complex LCEs with 3D deformations. In particular, 3D printing as an emerging technology to prepare LCEs allows to prepare sophisticated LCE structures. ${ }^{[44]}$ Nevertheless, the alignment direction is restricted, which depends on the extrusion direction of the LC mixture from the printer. Here, by combining plasma-induced polymerizations and magnetic field alignment we report on a new and simple strategy to achieve single-layer LCE bending actuation without the problem of delamination and without the need to use complex techniques of surface alignment. It is also a flexible strategy to obtain various deformation behaviors (bending and/or contraction) by adjusting the thickness of the LCE films or plasma powers. As examples of potential application, thermally actuated soft crawling walker and flower mimics were made by this LCE.

Plasma, which consists of a mixture of ions, electrons, radicals, excited species, photons, has attracted a considerable attention for surface cleaning, ${ }^{[45]}$ catalysis ${ }^{[46]}$ and polymerization. ${ }^{[47]}$ Low pressure plasma polymerization is considered as a common method to prepare polymer thin films with excellent mechanical properties and chemical stabilities. ${ }^{[48]}$ Gas is excited under vacuum conditions by supplying electrical energy and results in activated species, which initiate the surface polymerization to obtain thin cross-linked polymer films. Moreover, with the increase of electrical energy supplied, an increased electron density enhances the probability of molecules undergoing multiple electron impacts, ${ }^{[49]}$ which allows the preparation of polymer thin film with high crosslinking density.

Meanwhile, for people familiar with the plasma technology, a striking fact, which has been frequently overlooked, is that plasma generates lights of different wavelengths, ${ }^{[50]}$ which could be used to promote the photopolymerization of monomers. For example, Wertheimer et al ${ }^{[51]}$ reported the use of vacuum ultraviolet light (VUV, $\lambda=200 \mathrm{~nm}$ ) to carry out the 


\section{WILEY-VCH}

photopolymerization of amine-rich thin films from ammonia-hydrocarbon plasma gas mixtures. Nevertheless, the use of longer wavelengths $(\lambda=300-450 \mathrm{~nm})$ in the plasma for photopolymerization has not been reported. In this work, we combined the plasma-induced high crosslinking reactions on the surface and the plasma-induced photopolymerization $(\lambda=300-$ $450 \mathrm{~nm}$ ) in the bulk of LC monomers mixture to produce single-layer LCEs with bending deformation. Indeed, the plasma-induced high crosslinking reactions randomized the orientation of LC monomers aligned beforehand by magnetic field and resulted in a crosslinked surface without preferential alignment. Meanwhile the UV and visible light $(\lambda=300-450 \mathrm{~nm})$ generated by plasma penetrated the sample of LC monomers and polymerized them in the presence of photoinitiator to achieve aligned monodomain LC network. Consequently, the whole LCE sample presented a bending behavior under thermal stimulation. Moreover, by adjusting the supplied plasma power energies, the reaction times, and the sample thickness, LCE films with bending-contraction and only contraction behaviors were obtained. Owing to the simultaneous bending and contraction deformation in these LCE films, soft crawling walker and flower mimics were prepared. Finally, when LC monomers mixture was filled in a closed cell, the plasma-induced reactions could be suppressed and only plasma-induced photopolymerization operated to produce macro/micro-scale samples with contraction behavior. In brief, plasma-induced polymerizations provide a new and simple synthetic entry to LCE actuators with different deformation behaviors and various shapes and sizes.

\section{Results and discussion}

\subsection{Synthesis of the LC monomer and preparation of LCES}

The LC monomer C4 in Scheme 1 was synthesized according to Scheme S1. The details of syntheses and characterizations were described in the Supporting information.

The phase behavior of the LC monomer was studied by differential scanning calorimetry (DSC) and polarizing optical microscopy (POM). The LC monomer C4 exhibited two endothermic 


\section{WILEY-VCH}

transitions at 68 and $128{ }^{\circ} \mathrm{C}$ upon heating according to DSC measurement (Figure S3a). The observation by POM allowed to attribute these two transitions to crystal-to-nematic and nematic-to-isotropic phase transitions, respectively (Figure S3b). Upon cooling process, the LC monomer $\mathrm{C} 4$ exhibited a broad nematic phase from 126 to $20{ }^{\circ} \mathrm{C}$. For plasma-induced polymerizations, the LC monomers mixture was composed of LC monomer C4, chain-extender (2,2' -(ethylenedioxy)diethanethiol) (EDDET), LC crosslinker (RM82) and, when necessary, the photo-initiator 2-benzyl-2-(dimethylamino)-4'-morpholino butyrophenone (Iragure 369) (Scheme 1). The molar ratios of LC monomer, chain-extender, and LC crosslinker were kept at $\mathrm{C} 4:$ EDDET $: \mathrm{RM} 82=1: 1: 0.4$ in all the experiments. The concentration of the photoinitiator, when present, was kept at $2 \mathrm{wt} \%$. The phase transition of the LC monomers mixture (noted as LC mixture hereafter for simplicity) was checked beforehand to ensure that the mixture stayed in the nematic phase during the plasma-induced polymerizations at room temperature (RT). As shown in the DSC (Figure S4a), a phase transition appeared at $80^{\circ} \mathrm{C}$ during the cooling process, which was ascribed to the isotropic-nematic phase transition by POM. Interestingly, no recrystallization was observed upon cooling until RT. Moreover, recrystallization did not occur in the LC mixture left at RT for at least 4 days (see Figure S5 for DSC at different days after mixture preparation). Therefore, once the LC mixture was aligned beforehand, the LC alignment could be maintained during the vacuum process and the plasma process, which were performed at RT without intentional heating in the time scale of several hours. Besides, considering that the continued plasma treatment might increase the environment temperature, the temperature of the plasma chamber was monitored as a function of time under Argon plasma powers of $150 \mathrm{~W}$ and $200 \mathrm{~W}$, as shown in Figure S4b. Under 200 $\mathrm{W}$ and during $120 \mathrm{~min}$, which were the highest power and longest duration used in this research, the environmental temperature of the plasma could reach to $70{ }^{\circ} \mathrm{C}$ that was still lower than the nematic-isotropic phase transition of LC mixture. Therefore, the aligned nematic phase of LC 


\section{WILEY-VCH}

mixture was maintained during the used plasma process. This is a key point that enables to prepare aligned LCEs by plasma technique.

\subsection{LCEs prepared by plasma polymerization without photoinitiator}

Since the electrons, ions, and photons generated by plasma could initiate the polymerization, the plasma polymerization of the LC mixture was firstly investigated in the absence of photoinitiator (Figure 1a). The LC mixture was aligned in magnetic field as described in Experimental section below (see also Scheme S2a and 3a in Supporting Information). The aligned monomer mixture appeared almost transparent as observed by the naked eye (Figure 1b). Meanwhile, it exhibited transmittance variations observed by POM when changing the angle $\theta$ between its alignment direction and the $\mathrm{POM}$ polarizer direction from $0^{\circ}$ to $45^{\circ}$ (Figure S6a). Both observations indicated that the nematic phase of the LC mixture was well aligned. Then, a series of samples of the same aligned LC mixture were treated by plasmas of various power energies, i.e., $50 \mathrm{~W}, 100 \mathrm{~W}, 150 \mathrm{~W}$ and $200 \mathrm{~W}$, using Argon gas and during $30 \mathrm{~min}$. A white layer appeared on the surface of the film samples after the plasma polymerization (Figure 1c-d). POM observations showed that there were not clear light transmittance variations with the angle $\theta$ changing from $0^{\circ}$ to $45^{\circ}$ (Figure S6b-e). Therefore, the LC alignment of the sample at the surface has been disrupted during the plasma process. Other plasma parameters, such as pulsed plasma instead of continuous plasma, different gas $\left(\mathrm{N}_{2}\right.$ and $\left.\mathrm{O}_{2}\right)$ and different reaction time (120 min), were also used to polymerize the LC mixture. All samples showed similar results as observed by POM (Figure S7). Hence, the LC mixture without photoinitiator could not produce aligned LCEs by the plasma polymerization.

When immersing the above plasma-exposed films in dichloromethane, we noticed that a fraction of the material dissolved, but the upper white layer was insoluble. Thus, the plasma polymerization generated a crosslinked, but disorganized (non-aligned) layer at the sample surface, leaving unreacted the main body of the LC mixture. The gel content, i.e., the weight 


\section{WILEY-VCH}

ratio of crosslinked part versus the uncrosslinked part after plasma treatment was measured by gravimetry (see Experimental). The thickness of crosslinked part was then evaluated as 2-10 $\mu \mathrm{m}$, which increased with the increase of plasma power energy, reaching $\sim 10 \mu \mathrm{m}$ for a power of $200 \mathrm{~W}$ (Figure 1g).

\subsection{LCEs prepared in the presence of photoinitiator by plasma-induced polymerizations}

As described above, plasma polymerization in the absence of photoinitiator could only polymerize a thin layer $(2-10 \mu \mathrm{m})$ of monomer on the top of the sample. Then, lights of different wavelengths generated during the plasma process were examined, and the plasmainduced photopolymerization was attempted to polymerize/crosslink the LC mixture with photoinitiator added.

The spectra of the Argon plasma-generated lights at different plasma power energies are shown in Figure 2. Strong emissions in the near infrared (NIR) from $700-850 \mathrm{~nm}$ were detected, which could explain the moderate temperature elevation of plasma chamber described in the end of part 2.1. Emission peaks could also be observed between $300 \mathrm{~nm}$ to $450 \mathrm{~nm}$ (Figure 2a), which fall in the absorbance region of the photoinitiator Iragure 369. Moreover, the intensity of those peaks increased with the increase of plasma power (Figure 2b). Thus, the UV-Visible lights produced by the plasma could be used to initiate the photopolymerization of the LC mixtures in the presence of photoinitiator.

The polymerization of the aligned LC mixtures containing Iragure 369 was then carried out using plasma of different powers. The reaction time of 30 min was first used and the sample thickness was around $200 \mu \mathrm{m}$. After polymerization, the crosslinked gel contents of the final samples were estimated by gravimetry. The gel content was $15 \%$ and $56 \%$ for samples prepared with $50 \mathrm{~W}$ and $100 \mathrm{~W}$ plasma, respectively. That means $85 \%$ and $42 \%$ of starting materials were not crosslinked into LCEs (Table S1). In contrast, when the plasma power increased to $150 \mathrm{~W}$ and $200 \mathrm{~W}$, the gel content of the final sample were $69 \%, 71 \%$, respectively, which was 


\section{WILEY-VCH}

similar to gel contents of the LCEs prepared by UV polymerization for $30 \mathrm{~min}(73 \%) .{ }^{[52]}$ The relatively low gel content of this system was caused by the stoichiometric excess of allyl groups from both $\mathrm{C} 4$ and RM82 in the formulations (C4 : EDDET : RM82 = 1: 1: 0.4). RM82 participated in the elastomer as monomer and crosslinker, which might lead to unreacted allyl groups. The same level of gel contents were obtained by other groups ${ }^{[53],[54]}$ in similar LCEs prepared by photopolymerization, which exhibited stable reversible deformation upon stimulation. Thus, the plasma power of $150 \mathrm{~W}$ and $200 \mathrm{~W}$ and the reaction time of $30 \mathrm{~min}$ were sufficient here to conduct the plasma-induced photopolymerization of LC mixtures.

The deformation properties of LCE films with $150 \mathrm{~W}$ and $200 \mathrm{~W}$ plasma power were then investigated. Interestingly, the LCE film produced by $150 \mathrm{~W}$ plasma exhibited a reversible bending behavior upon heating and cooling (Figure 3a). In fact, a crosslinked surface part (face to plasma) without alignment was made by the normal plasma reactions, while an aligned LCE bulk part was formed by the photopolymerization. Therefore, in this single-layer LCE, the bulk part contracted along the direction of alignment, while the surface part did not contract. Consequently, the bending behavior was observed. Because the contraction part is much thicker $(\sim 190 \mu \mathrm{m})$ than the surface part $(\leq 10 \mu \mathrm{m})$, a shortening of the whole length was also obtained upon heating (Figure $\mathbf{3 b}, 150 \mathrm{~W}, 130^{\circ} \mathrm{C}$ ). Intriguingly, the LCE film produced by $200 \mathrm{~W}$ plasma showed only a reversible contraction behavior (Figure 3b). It was hypothesized that the photopolymerization might be faster than the plasma polymerization under $200 \mathrm{~W}$ plasma power. Thus, the whole sample was an aligned LCE obtained by photopolymerization. Indeed, POM image of LCE films prepared by $200 \mathrm{~W}$ plasma power confirmed the alignment of the whole sample (Figure S8). Moreover, a LCE film prepared by classical photopolymerization under $365 \mathrm{~nm}$ UV light was subsequently submitted to $200 \mathrm{~W}$ Argon plasma treatment for $30 \mathrm{~min}$. The final sample showed almost the same contraction behavior as before plasma treatment, indicating 200W plasma could hardly destroy the LCE surface alignment (Figure S9). 


\section{WILEY-VCH}

The evolutions of the bending deformation characterized by the curvature $1 / \mathrm{R}$ and of the contraction by length ratio $\mathrm{L} / \mathrm{L}_{\mathrm{iso}}$ of the $\mathrm{LCE}$ films as a function of temperature are shown in Figures 3c and 3d. For the LCE film made with $150 \mathrm{~W}$ plasma, upon heating the curvature remained near 0 when $\mathrm{T}<70{ }^{\circ} \mathrm{C}$, and increased rapidly for $\mathrm{T}$ increasing from 80 to $120{ }^{\circ} \mathrm{C}$. Upon cooling, the variation of the curvature was reversible with a slight hysteresis. On the other hand, the total length of the bent film at $130{ }^{\circ} \mathrm{C}$ was estimated as $4.3 \mathrm{~mm}$, relative to its initial length of $6 \mathrm{~mm}$ at RT (a 28\% of shortening took place, see Figure 3a). As for the LCE film made with $200 \mathrm{~W}$ plasma, in the whole temperature range from RT to $130{ }^{\circ} \mathrm{C}$ the LCE film shrank to half of its initial length at $\mathrm{RT}\left(\mathrm{L}_{\mathrm{RT}} / \mathrm{L}_{\mathrm{iso}}=2\right)$. Abrupt contraction took place upon heating in the temperature range $100-120^{\circ} \mathrm{C}$, around the nematic-isotropic transition temperature $\left(\mathrm{T}_{\mathrm{NI}}\right)$ of the LCE (Figure S10). The contraction and extension of LCE film were also reversible with a slight hysteresis. Figure $\mathbf{3 e}$ is the schematic illustration of the actuation of both LCE films.

Further, the reaction times were increased to $120 \mathrm{~min}$ to carry out the polymerization of the aligned LC mixtures containing Iragure 369 with plasma of different powers. Gel contents of the final sample increased: with 50W, 100W, $150 \mathrm{~W}$ and $200 \mathrm{~W}$ plasma, gel contents became $55 \%, 70 \%, 71 \%$ and $73 \%$, respectively (instead of $15 \%, 56 \%, 69 \%, 71 \%$ with reaction time of 30 min). The LCE films made with $100 \mathrm{~W}$ and $150 \mathrm{~W}$ plasma exhibited thermo-responsive reversible bending-contraction behaviors (Figure S11a and b), while the LCE film made with 200W plasma exhibited again thermo-responsive reversible contraction (Figures S11c). These results indicated that a low plasma power could also produce crosslinked LCE films with reaction time extension. Therefore, the deformation behaviors of the LCE films (bending or contraction) could be controlled by adjusting both the plasma power and the reaction time. The bending deformation $(1 / \mathrm{R})$ and the contraction/extension $\left(\mathrm{L} / \mathrm{L}_{\mathrm{iso}}\right)$ of the LCE film obtained under $150 \mathrm{~W}$ and 200W plasma powers and with two different reaction times ("plasma 30 min" 


\section{WILEY-VCH}

and "plasma 120 min") are summarized together in Figure S12. With longer reaction time, the deformation extent of the final sample increased.

Finally, the effect of the sample thickness on the deformation of LCEs prepared by plasmainduced polymerizations was studied. LCE films of thickness of 120, 190, 300, 380 and 450 $\mu \mathrm{m}$ were prepared with the plasma power of $150 \mathrm{~W}$ and the reaction time of $120 \mathrm{~min}$. As shown in Figure S13a-c, LCE films exhibited thermally actuated bending-contraction behaviors with the thickness of 120, 190 and $300 \mu \mathrm{m}$. However, with the thickness of 380 and $450 \mu \mathrm{m}, \mathrm{LCE}$ films showed only reversible contraction and extension (Figure S13d-e). Such sole contraction phenomena can be explained by the fact that the very thin crosslinked layer $(\sim 10 \mu \mathrm{m})$ generated by plasma reactions could not resist the contraction force of the thick part of LCEs $(>300 \mu \mathrm{m})$ upon heating process.

\subsection{LCEs prepared by plasma-induced photopolymerization without plasma contact}

As discussed above, the UV-Visible light generated by plasma did polymerize efficiently the LC mixture to prepare aligned LCEs. We then wondered if the UV-Visible light would still work when the plasma flux was not in direct contact with the monomer sample. In fact, the plasma needs to be in "touch" with the surface of the LC monomers mixture to induce any plasma reactions. To prevent the direct action of the plasma, a closed PDMS mold (Scheme S2b) was used to pack the LC mixture with a thickness of $160 \mu \mathrm{m}$. After exposing to a $150 \mathrm{~W}$ plasma during 120 min, the LCE film was removed from the PDMS mold. The LCE film contracted to half of its initial length at $\mathrm{RT}\left(\mathrm{L}_{\mathrm{RT}} / \mathrm{L}_{\mathrm{iso}}=2\right)$ when heated to isotropic phase as shown in Figure $\mathbf{4 b}$. Therefore, it was confirmed definitively that the light produced by the plasma was able to induce the photopolymerization of the LC monomers.

By making use of this close system, LCE micropillars of $100 \mu \mathrm{m}$ height and $20 \mu \mathrm{m}$ diameter $(\mathrm{H} 100 \mu \mathrm{m} \times \mathbf{x} 20 \mu \mathrm{m})$ as shown in Figure $\mathbf{4 c}$ were also prepared by plasma-induced photopolymerization with the same alignment setup developed previously ${ }^{[28,55]}$ (see also 


\section{WILEY-VCH}

Experimental section and Scheme S2c and S3c). The aligned LC mixture in the closed PDMS micropillar mold was put in the plasma chamber and polymerized under $150 \mathrm{~W}$ plasma during $120 \mathrm{~min}$. The obtained micropillars $(\mathrm{H} 100 \mu \mathrm{m} \times \Phi 20 \mu \mathrm{m})$ cut from the sample exhibited typical reversible contraction-extension with temperature changes. Thermal stability was tested by 10 heating-cooling cycles (Figure S14), showing no change in contraction ratio.

\subsection{Soft crawling walker and flower mimics}

Taking advantage of the simultaneous bending and contraction deformation of LCEs described above, we have made a soft crawling walker that mimicked the motion of caterpillar. As shown in Figure 5a, a LCE film with thermally activated bending-contraction behavior (length 4.5 mm, width $1.5 \mathrm{~mm}$; thickness: $\sim 300 \mu \mathrm{m}$ ) was placed on a surface with micro-ratchets roughness. The LCE film was prepared with $150 \mathrm{~W}$ Argon plasma during $120 \mathrm{~min}$. Stimulated by heatingcooling cycles, the LCE film moved along the surface as shown in Figure $\mathbf{5 b}$. The film bended and contracted on heating, while on cooling the position return of the left side of the LCE due to the extension was blocked by the steep side of one of the ratchet teeth (Figure S15b). Consequently, the bending film extended towards the smooth side of the teeth and a forward motion in right direction was obtained along the surface. As shown in Figure 5c, after five successive heating and cooling cycles, the LCE film moved forward about $7 \mathrm{~mm}$. On average, LCE film moved $30 \%$ of its original length in each heating-cooling cycle.

In addition, a flower mimic was also prepared with a cross-shaped LCE sample as shown in Figure 5d and 5e. The sample was prepared with homeotropic alignment (see Experimental and Scheme S2d and S3d) and polymerized with 150W Argon plasma during $120 \mathrm{~min}$. The cross-shaped LCE was composed of a crosslinked surface without alignment and a homeotropically aligned bulk (Figure 5d). Upon heating, the bulk part of cross-shaped LCE expanded because of the contraction along the thickness direction, while the surface part maintained the same size. Consequently, the sample curved up like the closure of a four-petal flower (Figure 5e). Upon cooling, the flower mimicking cross-shaped LCE re-opened. 


\section{WILEY-VCH}

\section{Conclusions}

Main-chain nematic liquid crystal elastomers (LCEs) were successfully prepared by plasmainduced polymerizations. Two distinct reactions were identified, plasma polymerization and plasma-induced photopolymerization (in the presence of photoinitiator). The plasma polymerization formed crosslinked but disoriented structures at the surface of the film sample, while the plasma-induced photopolymerization produced aligned LCE structure from the remaining monomers in the bulk. The actuation behaviors of these single-layer LCE films could be adjusted by the plasma power, the reaction time, and the sample thickness. Typically, with plasma power of $150 \mathrm{~W}$, reaction time of $30 \mathrm{~min}$ or $120 \mathrm{~min}$, and sample thickness smaller than $300 \mu \mathrm{m}$, thermally activated simultaneous bending and contraction could be obtained in these single-layer LCEs. The bending deformation was caused by the contraction of the bulk part along the direction of alignment and the non-contraction of the surface part face to the plasma flux. On the other hand, only contraction behavior was observed when LCE sample had a thickness superior to $300 \mu \mathrm{m}$ or was polymerized with high plasma power (200W). Moreover, it was demonstrated that the light generated by the plasma could photopolymerize alone the LC mixtures packed in the closed cells, where the plasma flux was not in contact with monomers, to obtain macroscopic LCE films and microscopic micropillars with reversible contractionextension behavior. Finally, the LCE films with simultaneous bending and contraction actuation were used to construct a soft crawling walker that could move along the surface with microratchet roughness upon heating-cooling cycles and a flower mimic that could close and open with temperature change.

Plasma technology is enriching the preparation methods available for LCEs and shows advantages in the preparation of single-layer LCEs with bending and contraction actuation.

\section{Experimental section}

\subsection{Materials and characterization}




\section{WILEY-VCH}

Allyl alcohol (98\%), potassium carbonate $\left(\mathrm{K}_{2} \mathrm{CO}_{3}\right)$, sodium hydroxide $(\mathrm{NaOH})$ were purchased from Alfa-Aesar. Ethyl 4-hydroxybenzoate (98\%), sodium hydride (90\%), N,N`dicyclohexylcarbodiimide (99\%), 4-pyrrolidinopyridine( 98\%), methylhydroquinone (99\%), (ethylenedioxy) diethanethiol (EDDET, 95\%) and Irgacure 369 (2-benzyl-2-(dimethylamino)4'-morpholinobutyrophenone) (97\%) were purchased from Sigma-Adrich. All these compounds were used as received without purification. 2-Methyl-1,4-phenylene bis(4-((6(acryloyloxy)hexyl)oxy)benzoate) (RM82, 95\%) was purchased from Hunan Huateng Pharmaceutical Co., Ltd in China and recrystallized in ethanol before using. Solvents like tetrahydrofuran (THF), dichloromethane (DCM), petroleum ether $\left(\mathrm{PE}, 40-60^{\circ} \mathrm{C}\right)$ and acetone with analytical pure grade were obtained from VWR and used without purification.

${ }^{1} \mathrm{H}$ NMR and ${ }^{13} \mathrm{C}$ NMR spectra were recorded on a Bruker Avance $400 \mathrm{MHz}$ spectrometer at 297 K. Deuterated chloroform $\left(\mathrm{CDCl}_{3}\right)$ was used as the solvent and TMS (tetramethylsilane) as the internal reference. Calorimetric measurements of LC monomers and elastomers were performed using a PerkinElmer DSC7 instrument. LC monomer or monomer mixture were scanned over a temperature range between $-40{ }^{\circ} \mathrm{C}$ and $120{ }^{\circ} \mathrm{C}$ at a rate of $5{ }^{\circ} \mathrm{C} / \mathrm{min}$. LCE samples were scanned over a temperature range between $-40{ }^{\circ} \mathrm{C}$ and $150{ }^{\circ} \mathrm{C}$ at $10{ }^{\circ} \mathrm{C} / \mathrm{min}$. Polarized Optical microscopy (POM) observations were carried out on a Motic BA310POL equipped with a Motic CCD camera and a Mettler FP 80 hot stage. The emission spectra of the plasma were measured with a detector SpectraPro 2750. The low-pressure plasma polymerization was done using the Flarion Series FLR300 (13.56MHz-300 W) apparatus

provided by Plasmionique (Québec, Canada) ${ }^{[56-58]}$ equipped with a two-stage rotary vacuum pump. The images were captured by the camera of a smartphone fixed on a tripod.

\subsection{Alignment method for the LC monomer mixtures}

The different molds and alignment processes are shown in Scheme S2 and S3.

For planar alignment of film samples, the LC mixture, containing the LC monomer C4, the chain-extender EDDET, the LC crosslinker RM82 and, if needed, the photo-initiator Iragure 


\section{WILEY-VCH}

369, was added into the rectangular hole of a PDMS mold placed on a glass slide, the common size of the rectangular hole being $\sim 6 \mathrm{~mm} \times 2 \mathrm{~mm} \times \sim 200 \mu \mathrm{m}$ (length $\times$ width $\times$ thickness) (Scheme S2a). There were two kinds of molds: closed mold and open mold. To fill uniformly the mold, the LC mixture was heated up to its isotropic phase $\left(110^{\circ} \mathrm{C}\right)$ and added into the mold progressively. Then, the LC mixture-filled mold was placed at the center of a holder equipped with two permanent magnets facing each-other (1 T NdFeB rare earth magnet) (Scheme S3a and $\mathbf{S 3 b})$. A slow cooling $\left(1{ }^{\circ} \mathrm{C} / \mathrm{min}\right)$ from isotropic phase to room temperature in the presence of the magnetic field resulted in the planar alignment of the nematic phase in the LC mixture. After alignment the LC mixture-filled mold was moved to the plasma chamber.

For micropillars preparation, the LC mixture was deposited on a glass coverslip placed atop a permanent magnet ( $\sim 1 \mathrm{~T} \mathrm{NdFeB}$ rare earth magnet) in a heating stage (Scheme S3c). The mixture was first heated up to its isotropic phase $\left(110^{\circ} \mathrm{C}\right)$. A soft PDMS mold with cylindrical holes $(\mathrm{H} 100 \mu \mathrm{m} \times \Phi 20 \mu \mathrm{m})$ was gently pressed onto the melted mixture and then cooled down to room temperature at a slow rate $\left(1^{\circ} \mathrm{C} / \mathrm{min}\right)$. During the cooling process, the magnetic field aligned the nematic phase with the nematic director along the long axis of the cylinder holes.

For the cross-shaped LCE, LC mixture was filled into a cross-shaped hole with four arms, the size of each arm being $\sim 6 \mathrm{~mm} \times \sim 2 \mathrm{~mm} \times \sim 200 \mu \mathrm{m}$ (length $\mathrm{x}$ width $\mathrm{x}$ thickness). The alignment setup was shown in the Scheme S3d where a homeotropic alignment was produced. The same alignment process was carried out as that described for planar alignment described above.

\subsection{Low-pressure plasma polymerization process}

The aligned LC mixture with its mold was put inside the low-pressure plasma device. Turbomolecular pump backed by a two-stage rotary vane pump was used to evacuate the air from the chamber to a base pressure of $<10^{-6}$ mbar. Then, Argon gas was introduced into the chamber until the chamber pressure reached $10^{-1}$ mbar. At last, the plasma power energy and the reaction 


\section{WILEY-VCH}

time were adjusted according to the conditions chosen. After polymerization, the LCE film was recovered by removing it from the mold.

\subsection{Gel content measurement by gravimetry}

After polymerization, the crosslinked gel contents of the final samples were estimated by gravimetry as follows. The polymerized sample was immersed in dichloromethane for $30 \mathrm{~min}$ to solubilize the unreacted monomers and un-crosslinked polymers, and the residues were dried overnight in vacuum at $70{ }^{\circ} \mathrm{C}$. The weight of the dried residue $\left(\mathrm{m}_{\mathrm{r}}\right)$ over that of the initial sample $\left(\mathrm{m}_{0}\right)$ gave the gel content $\left(\mathrm{m}_{\mathrm{r}} / \mathrm{m}_{0}\right)$.

\section{Conflicts of interest}

There are no conflicts to declare.

\section{Supporting Information}

Supporting Information is available from the Wiley Online Library or from the author.

\section{Acknowledgements}

This work has received the support from the French National Research Agency (ANR-16CE29-0028) and from the "Institut Pierre-Gilles de Gennes" (IPGG, laboratoire d'excellence, “Investissements d'avenir” programs ANR-10-IDEX-0001-02 PSL, ANR10-LABX-31 and ANR-10-EQPX-34). Bin NI gratefully acknowledges the China Scholarship Council for funding his Ph.D. scholarship. We would like to thank Prof. Axel Bugin from Sorbonne University and Institut Curie for fruitful discussions. Mr. Rémy Fert and Mr. Eric Nicolau in the mechanical workshop of UMR168 CNRS in Institut Curie are acknowledged for building the setup used in the alignment by two magnets.

Received: ((will be filled in by the editorial staff))

Revised: ((will be filled in by the editorial staff)) Published online: ((will be filled in by the editorial staff)) 


\section{WILEY-VCH}

\section{References}

[1] L. Hines, K. Petersen, G. Z. Lum, M. Sitti, Adv. Mater. 2017, 29, 1603483.

[2] J. Kim, J. W. Kim, H. C. Kim, L. Zhai, H.-U. Ko, R. M. Muthoka, Int. J. Precis. Eng. Manuf. 2019, 20, 2221.

[3] M.-H. Li, P. Keller, B. Li, X. Wang, M. Brunet, Adv. Mater. 2003, 15, 569.

[4] Y. Yu, M. Nakano, T. Ikeda, Nature 2003, 425, 145.

[5] C. Ohm, M. Brehmer, R. Zentel, Adv. Mater. 2010, 22, 3366.

[6] R. S. Kularatne, H. Kim, J. M. Boothby, T. H. Ware, J. Polym. Sci., Part B: Polym. Phys. 2017, 55, 395 .

[7] Y. Shang, J. Wang, T. Ikeda, L. Jiang, J. Mater. Chem. C 2019, 7, 3413.

[8] M.-H. Li, P. Keller, J. Yang, P.-A. Albouy, Adv. Mater. 2004, 16, 1922.

[9] M. Wang, B.-P. Lin, H. Yang, Nat. Comm. 2016, 7, 13981.

[10] A. H. Gelebart, D. Jan Mulder, M. Varga, A. Konya, G. Vantomme, E. W. Meijer, R. L. B. Selinger, D. J. Broer, Nature 2017, 546, 632.

[11] T. H. Ware, M. E. McConney, J. J. Wie, V. P. Tondiglia, T. J. White, Science 2015, 347, 982.

[12] C. Ohm, C. Serra, R. Zentel, Adv. Mater. 2009, 21, 4859.

[13] Y. Yang, F. Ma, Z. Li, J. Qiao, Y. Wei, Y. Ji, J. Mater. Chem. A 2017, 5, 7285.

[14] P.-G. D. Gennes, Comptes Rendus Hebd. Seances. Acad. Sci. Ser. B 1975, 101.

[15] H.-F. Lu, M. Wang, X.-M. Chen, B.-P. Lin, H. Yang, J. Am. Chem. Soc. 2019, 141, 14364.

[16] L. Liu, M.-H. Liu, L.-L. Deng, B.-P. Lin, H. Yang, J. Am. Chem. Soc. 2017, 139, 11333.

[17] F. Cheng, R. Yin, Y. Zhang, C.-C. Yen, Y. Yu, Soft Matter 2010, 6, 3447.

[18] O. M. Wani, H. Zeng, A. Priimagi, Nat. Commun. 2017, 8, 15546.

[19] Y. Liu, B. Xu, S. Sun, J. Wei, L. Wu, Y. Yu, Adv. Mater. 2017, 29, 1604792.

[20] C.-K. Chang, C. M. W. Bastiaansen, D. J. Broer, H.-L. Kuo, Adv. Funct. Mater. 2012, 22, 2855. 


\section{WILEY-VCH}

[21] M. Yamada, M. Kondo, J.-i. Mamiya, Y. Yu, M. Kinoshita, C. J. Barrett, T. Ikeda, Angew. Chem. Int. Ed. 2008, 47, 4986.

[22] Z.-C. Jiang, Y.-Y. Xiao, X. Tong, Y. Zhao, Angew. Chem. Int. Ed. 2019, 58, 5332.

[23] H. Zeng, O. M. Wani, P. Wasylczyk, R. Kaczmarek, A. Priimagi, Adv. Mater. 2017, 29, 1701814.

[24] L. Dong, Y. Zhao, Mater. Chem. Front. 2018, 2, 1932.

[25] J. Küpfer, H. Finkelmann, Makromo. Chem. , Rapid Commun. 1991, 12, 717.

[26] D. J. Broer, G. N. Mol, G. Challa, Die Makromolekulare Chemie 1989, 190, 19.

[27] D. L. Thomsen, P. Keller, J. Naciri, R. Pink, H. Jeon, D. Shenoy, B. R. Ratna, Macromolecules 2001, 34, 5868.

[28] A. Buguin, M.-H. Li, P. Silberzan, B. Ladoux, P. Keller, J. Am. Chem. Soc. 2006, 128, 1088.

[29] L. T. De Haan, C. Sánchez-Somolinos, C. M. W. Bastiaansen, A. P. H. J. Schenning, D. J. Broer, Angew. Chem. Int. Ed. 2012, 51, 12469.

[30] S.-k. Ahn, T. H. Ware, K. M. Lee, V. P. Tondiglia, T. J. White, Adv. Funct. Mater. 2016, 26,5819 .

[31] Y. Xia, G. Cedillo-Servin, R. D. Kamien, S. Yang, Adv. Mater. 2016, 28, 9637.

[32] Y. Xia, X. Zhang, S. Yang, Angew. Chem. Int. Ed. 2018, 57, 5665.

[33] Y. Naka, J.-i. Mamiya, A. Shishido, M. Washio, T. Ikeda, J. Mater. Chem. 2011, 21, 1681.

[34] Q. Qian, J. Chen, M.-H. Li, P. Keller, D. He, J. Mater. Chem. 2012, 22, 4669.

[35] J. M. Boothby, T. H. Ware, Soft Matter 2017, 13, 4349.

[36] J. Hu, X. Li, Y. Ni, S. Ma, H. Yu, J. Mater. Chem. C 2018, 6, 10815.

[37] H. Kim, J. A. Lee, C. P. Ambulo, H. B. Lee, S. H. Kim, V. V. Naik, C. S. Haines, A. E. Aliev, R. Ovalle-Robles, R. H. Baughman, T. H. Ware, Adv. Funct. Mater. 2019, 29, 1905063. [38] G. N. Mol, K. D. Harris, C. W. M. Bastiaansen, D. J. Broer, Adv. Funct. Mater. 2005, 15, 1155. 


\section{WILEY-VCH}

[39] C. Zhang, X. Lu, G. Fei, Z. Wang, H. Xia, Y. Zhao, ACS Appl. Mater. Interfaces 2019, 11, 44774.

[40] J. Hu, Z.-Y. Kuang, L. Tao, Y.-F. Huang, Q. Wang, H.-L. Xie, J.-R. Yin, E.-Q. Chen, ACS Appl. Mater. Interfaces 2019, 11, 48393.

[41] M. O. Saed, C. P. Ambulo, H. Kim, R. De, V. Raval, K. Searles, D. A. Siddiqui, J. M. O.

Cue, M. C. Stefan, M. R. Shankar, T. H. Ware, Adv. Funct. Mater. 2019, 29, 1806412.

[42] S. J. Aßhoff, F. Lancia, S. Iamsaard, B. Matt, T. Kudernac, S. P. Fletcher, N. Katsonis, Angew. Chem. Int. Ed. 2017, 56, 3261.

[43] L. Liu, B. Geng, S. M. Sayed, B.-P. Lin, P. Keller, X.-Q. Zhang, Y. Sun, H. Yang, Chem. Commun. 2017, 53, 1844.

[44] C. P. Ambulo, J. J. Burroughs, J. M. Boothby, H. Kim, M. R. Shankar, T. H. Ware, ACS Appl. Mater. Interfaces 2017, 9, 37332.

[45] B. O. Aronsson, J. Lausmaa, B. Kasemo, J. Biomed. Mater. Res. 1997, 35, 49.

[46] T. Nozaki, K. Okazaki, Catal. Today 2013, 211, 29.

[47] H. Yasuda, Plasma polymerization, Academic Press, New York 1985.

[48] D. Thiry, S. Konstantinidis, J. Cornil, R. Snyders, Thin Solid Films 2016, 606, 19.

[49] J. Ryssy, E. Prioste-Amaral, D. F. N. Assuncao, N. Rogers, G. T. S. Kirby, L. E. Smith, A. Michelmore, Phys. Chem. Chem. Phys. 2016, 18, 4496.

[50] L. Potočňáková, J. Hnilica, V. Kudrle, Open Chem. 2014, 13, 577

[51] F. Truica-Marasescu, J.-C. Ruiz, M. R. Wertheimer, Plasma Process. Polym. 2012, 9, 473.

[52] I. De Bellis, B. Ni, D. Martella, C. Parmeggiani, P. Keller, D. S. Wiersma, M.-H. Li, S. Nocentini, Adv. Intell. Sys. 2020, 2000035.

[53] T. H. Ware, Z. P. Perry, C. M. Middleton, S. T. Iacono, T. J. White, ACS Macro Lett. 2015, 4, 942 .

[54] T. Hessberger, L. Braun, R. Zentel, Polymers 2016, 8, 410. 
[55] B. Ni, H. Chen, M. Zhang, P. Keller, M. Tatoulian, M.-H. Li, Mater. Chem. Front. 2019, 3, 2499.

[56] Y. Ladner, F. D’Orlyé, C. Perréard, B. Da Silva, C. Guyon, M. Tatoulian, S. Griveau, F. Bedioui, A. Varenne, Plasma Process. Polym. 2013, 10, 959.

[57] Y. Ladner, F. D’Orlyé, C. Perréard, B. Da Silva, C. Guyon, M. Tatoulian, S. Griveau, F. Bedioui, A. Varenne, Plasma Process. Polym. 2014, 11, 518.

[58] T. Roques-Carmes, H. Semara, C. Guyon, M. Tatoulian, S. Ognier, J.-M. Commenge, Chem. Eng. Sci. 2014, 118, 245. 


\section{WILEY-VCH}

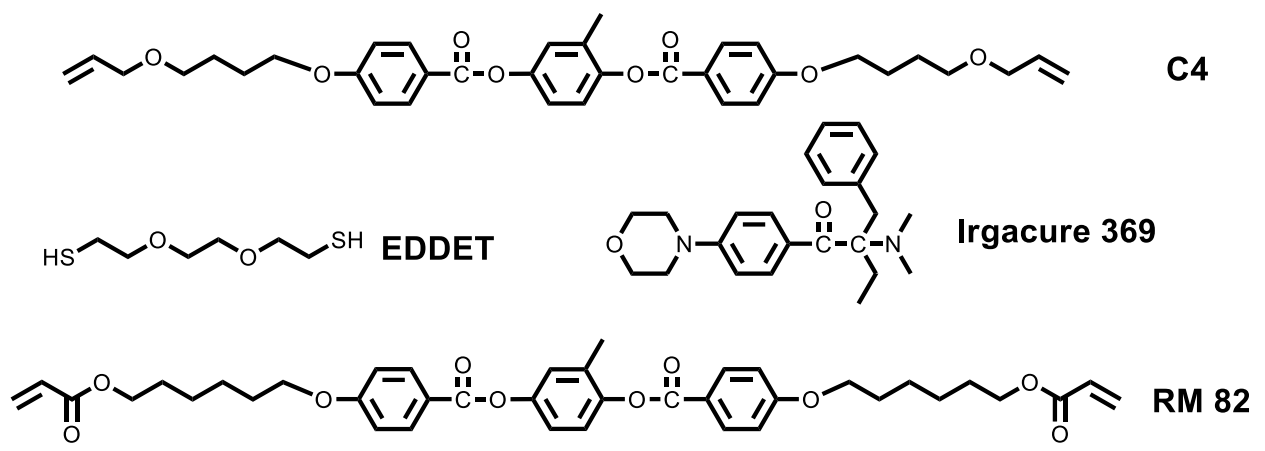

Scheme 1. Molecular structures of the components in the LC monomers mixture.
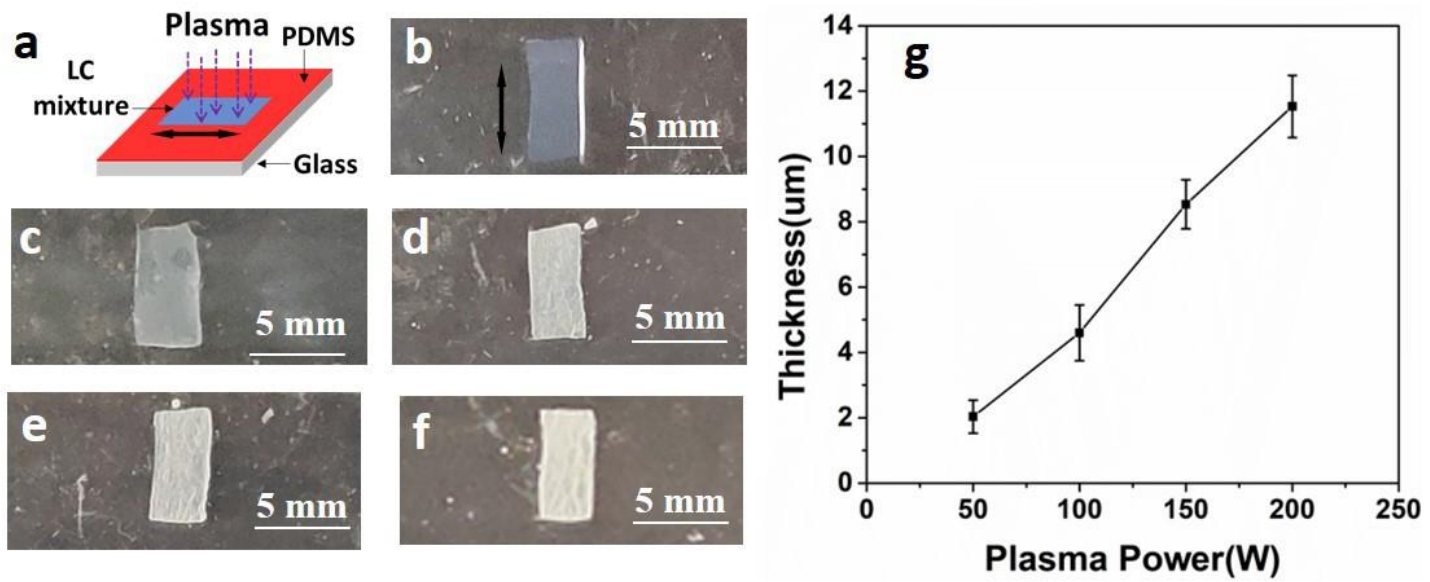

Figure 1. (a) Schematic of "open" mold for LCE preparation via plasma polymerization. (b-f) Photographs of the sample surfaces before (b) and after Argon plasma polymerization with plasma power of $50 \mathrm{~W}$ (c), $100 \mathrm{~W}$ (d), $150 \mathrm{~W}$ (e) and $200 \mathrm{~W}$ (f), respectively. (g) The thickness of polymerized LCE upper layer under different plasma energies. Argon flow rate was fixed at $50 \mathrm{~mL} / \mathrm{min}$, the reaction time as $30 \mathrm{~min}$. The double arrow represents the orientation direction of nematic director in LC mixture.
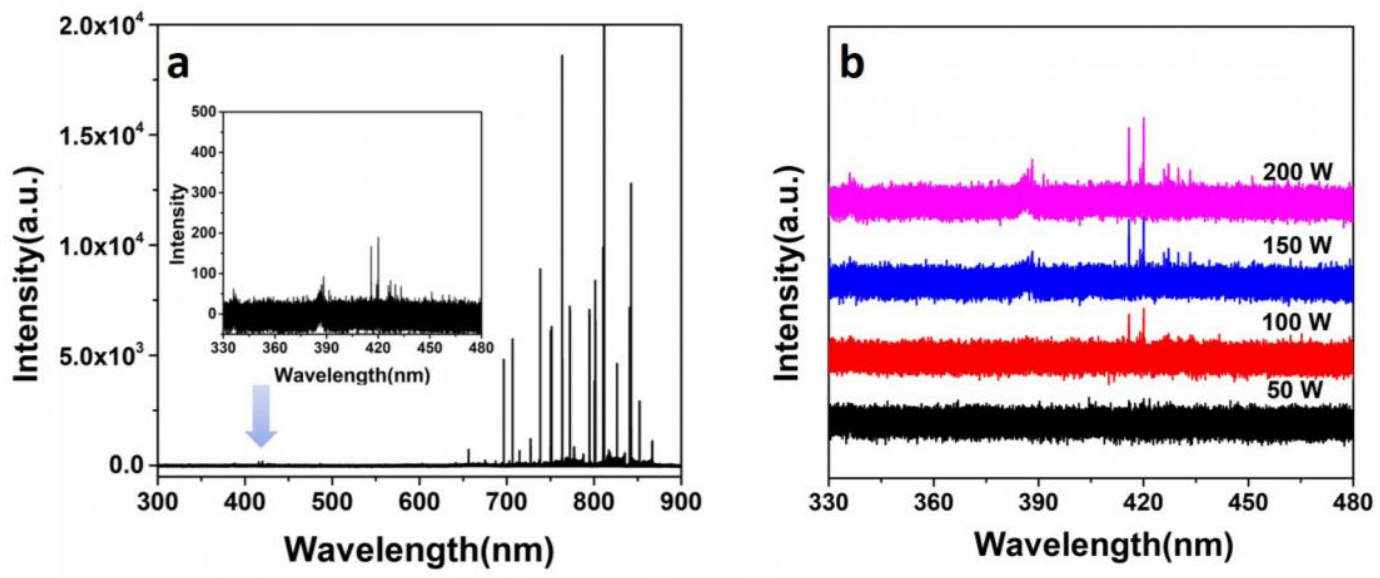

Figure 2. (a) Light emission spectra of the Argon plasma at $200 \mathrm{~W}$. (b) Light emission spectra of the Argon plasma of different powers in UV-Visible region. 


\section{WILEY-VCH}

a
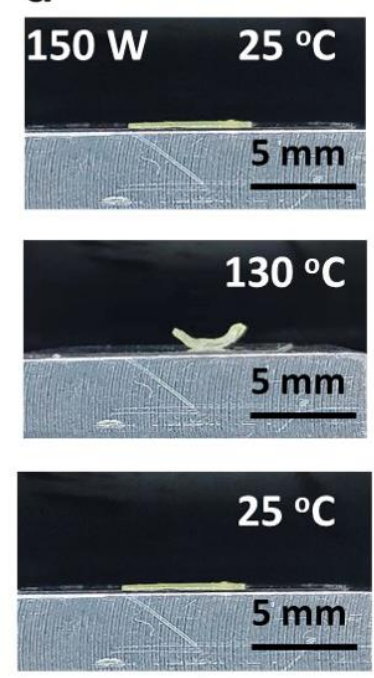

e

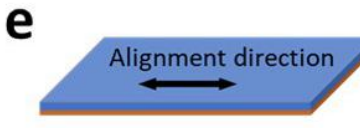

$150 \mathrm{~W}$

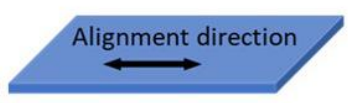

$200 \mathrm{~W}$ b
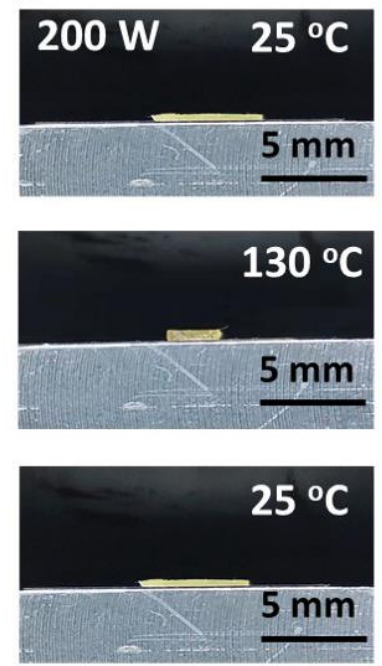

C

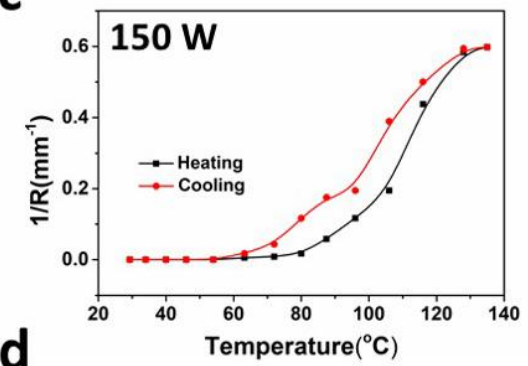

d

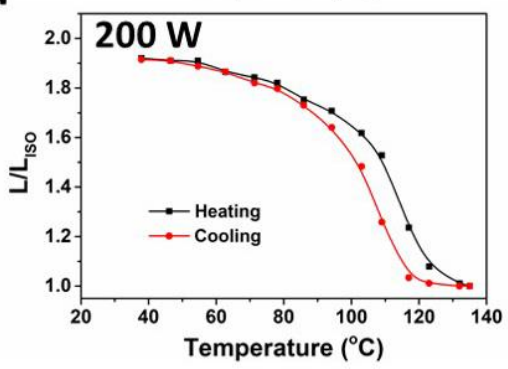

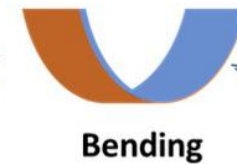

Cooling

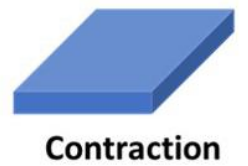

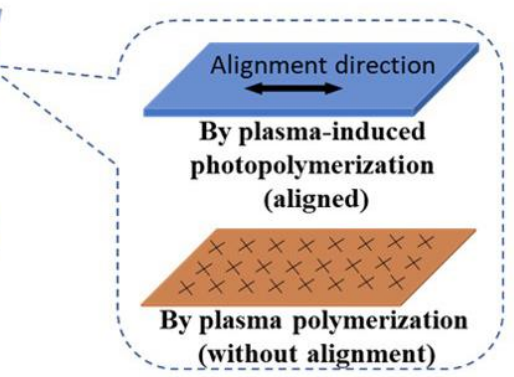

Figure 3. (a) and (b) The deformation behaviors of the LCE films fabricated by plasma-induced polymerizations with $150 \mathrm{~W}$ and $200 \mathrm{~W}$ plasma powers during $30 \mathrm{~min}$ and in the presence of photoinitiator. The surface part face to plasma of the sample was put down on the hot stage (gray blocks in the photos). (c) The curvature (1/R) of the LCE film polymerized by $150 \mathrm{~W}$ plasma as a function of temperature upon heating and cooling. $\mathrm{R}$ is the radius of the curved film. (d) The contraction and extension of the LCE film polymerized by $200 \mathrm{~W}$ plasma upon heating and cooling, represented by $\mathrm{L} / \mathrm{L}_{\text {iso }}$ as a function of temperature. $\mathrm{L}$ and $\mathrm{L}_{\text {iso }}$ are the lengths of the LCE film along the netmatic alignment at a given temperature $\mathrm{T}$ and in its isotropic state, respectively. (e) Schematic illustration of the bending and contraction behavior of the LCE films. 


\section{WILEY-VCH}

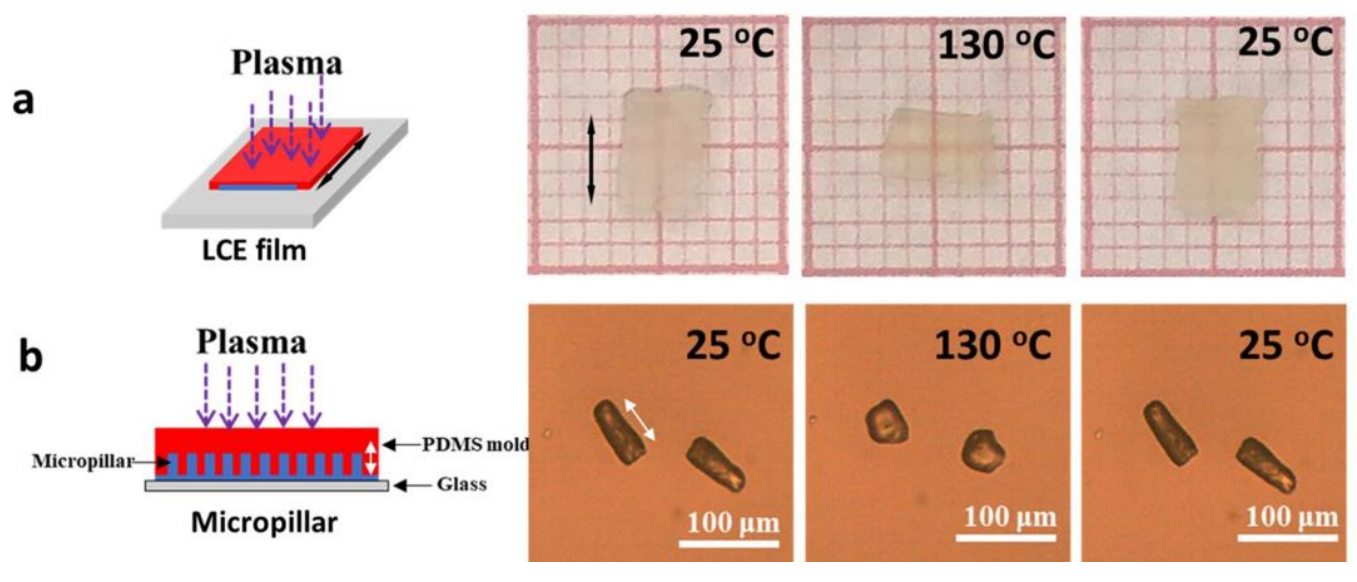

Figure 4. Thermo-responsive deformation of the LCE polymerized in the closed PDMS mold only by the light produced from the $150 \mathrm{~W}$ plasma for $120 \mathrm{~min}$. (a) Macroscopic LCE film sample: preparation (left) and their size upon heating and cooling at $25^{\circ} \mathrm{C}, 130^{\circ} \mathrm{C}$ at $25^{\circ} \mathrm{C}$ (right). (b) Micro-pillars sample: preparation (left) and the sizes of the cut pillars upon heating and cooling at $25^{\circ} \mathrm{C}, 130^{\circ} \mathrm{C}$ at $25^{\circ} \mathrm{C}$ (right). The alignment direction of the nematic phase is indicated by black double arrow in (a) and by write double arrow in (b). 


\section{WILEY-VCH}
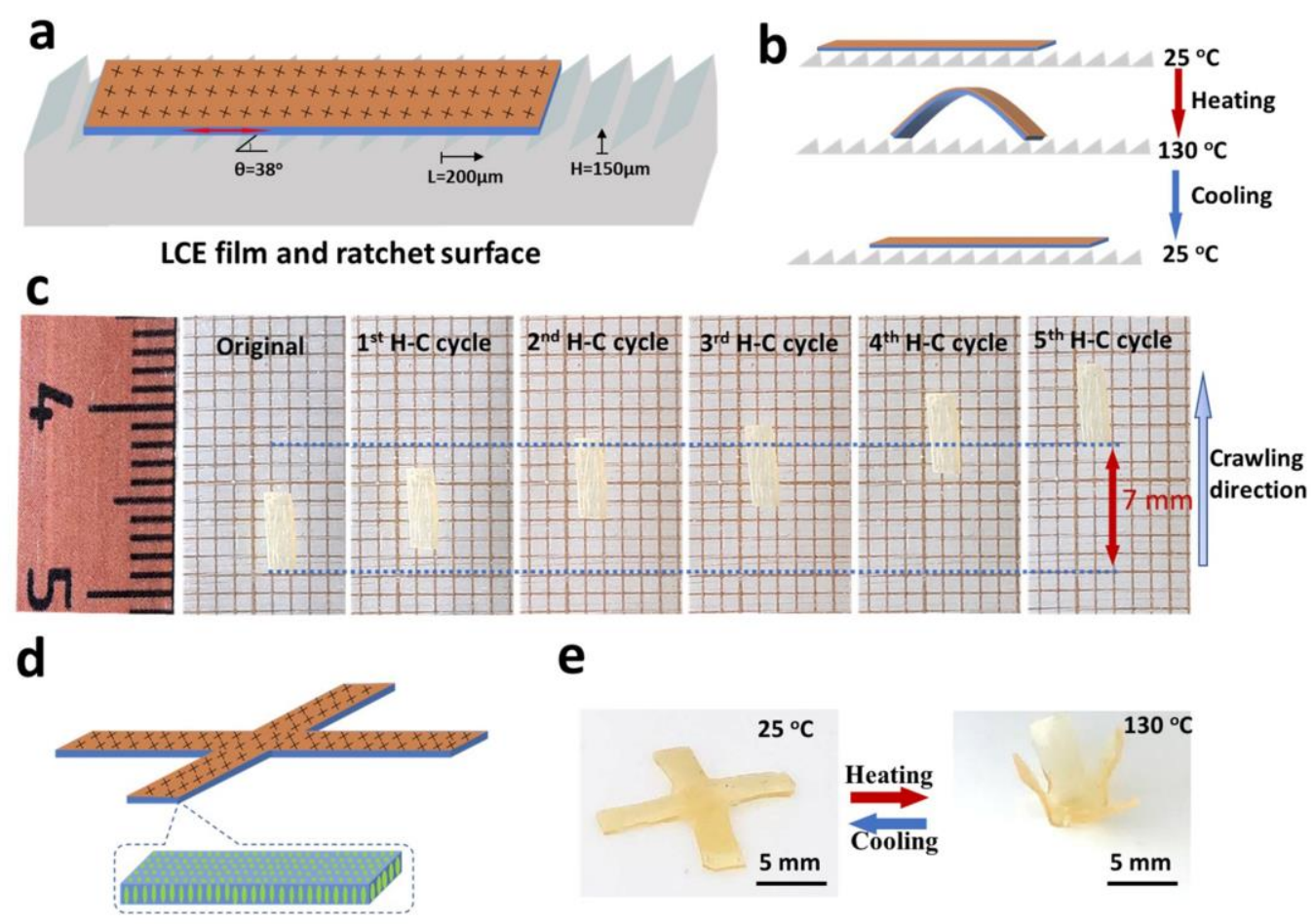

e

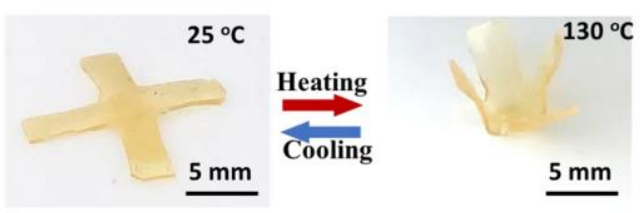

Figure 5. (a) Schematic presentation of the LCE film on the ratchet surface. LCE was prepared by plasma-induced polymerizations with non-aligned surface part (brown) and planarly aligned bulk part (blue). (b) Locomotion illustration of LCE film with bending-contraction behaviors on a surface with micro-ratchet roughness. (c) Photographs showing the displacements of the LCE film on the ratchet surface made of PDMS upon several heating-cooling $(\mathrm{H}-\mathrm{C})$ cycles. The rachet surface was put on a glass slide, under which a grid paper was placed for position measurement. Both ratchet surface and glass slide are transparent and not visible in the picture. (d) Schematic presentation of the cross-shaped LCE prepared by plasma-induced polymerizations with non-aligned surface part (brown) and homeotropically aligned bulk part (blue). The size of each of four petals is $6 \mathrm{~mm} \times 1.5 \mathrm{~mm} \mathrm{x} 200 \mu \mathrm{m}$ (length $\mathrm{x}$ width $\mathrm{x}$ thickness). (e) Cross-shaped LCE exhibits flower-like opening and closing upon thermal stimulation. 


\section{WILEY-VCH}

A new strategy with plasma technology was developed to prepare single-layer main-chain LCEs that exhibited thermally actuated bending and contraction deformations, from which soft robots like crawling walker and flower mimic were built.

Bin Ni, ${ }^{a}$ Mengxue Zhang, ${ }^{a}$ Cedric Guyon, ${ }^{a}$ Patrick Keller,$*{ }^{*}$ Michael Tatoulian, ${ }^{*},{ }^{a}$ and Min-Hui $\mathrm{Li}^{*}{ }^{a}$

Plasma-induced polymerizations: a new synthetic entry in liquid crystal elastomer actuators

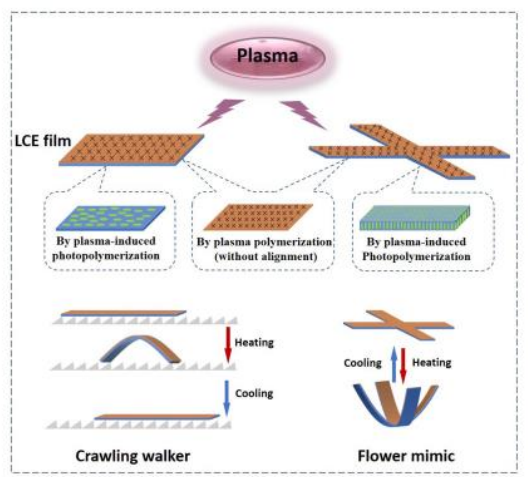

